Philosophie ANTIQUE

\section{Philosophie antique}

Problèmes, Renaissances, Usages

$14 \mid 2014$

Le devoir

\title{
Le propylée et la statue
}

Présence et absence du néoplatonisme dans la lecture foucaldienne de la philosophie antique

Isabelle Koch

\section{(2) OpenEdition}

1 Journals

Édition électronique

URL : https://journals.openedition.org/philosant/816

DOI : 10.4000/philosant.816

ISSN : 2648-2789

Éditeur

Éditions Vrin

\section{Édition imprimée}

Date de publication : 1 novembre 2014

Pagination : 291-319

ISBN : 978-2-7574-0855-1

ISSN : 1634-4561

\section{Référence électronique}

Isabelle Koch, « Le propylée et la statue », Philosophie antique [En ligne], 14 | 2014, mis en ligne le 01 novembre 2018, consulté le 03 décembre 2022. URL : http://journals.openedition.org/philosant/816 DOI : https://doi.org/10.4000/philosant.816

\section{(c) (i) $\odot$}

Creative Commons - Attribution - Pas d'Utilisation Commerciale - Pas de Modification 4.0 International - CC BY-NC-ND 4.0

https://creativecommons.org/licenses/by-nc-nd/4.0/ 


\section{LE PROPYLÉE ET LA STATUE \\ Présence et absence du néoplatonisme dans la lecture foucaldienne de la philosophie antique Isabelle $\mathrm{KOCH}$}

Aix Marseille Université, IHP EA 3276

13621, Aix-en-Provence, France

RÉSUMÉ. L'interprétation de l'Antiquité gréco-latine que Michel Foucault développe dans ses derniers ouvrages publiés et ses derniers cours au Collège de France fait peu de place au néoplatonisme. L'Herméneutique du sujet, seul texte où il en soit question avec quelque attention, le présente comme un simple prolongement de la philosophie de Platon, relevant du «modèle platonicien » du souci de soi, que Foucault confronte aux modèles hellénistico-romain et chrétien. Pourtant, c'est chez les commentateurs néoplatoniciens de l'Alcibiade que Foucault trouve certaines distinctions, en particulier entre « politique » et « cathartique », qui fondent la construction de ces trois modèles et leur articulation. Ce rôle architectonique des néoplatoniciens tardifs, par ailleurs, va de pair avec l'absence quasi totale d'un autre néoplatonisme, celui de Plotin, qui pourtant semblerait à première vue mieux trouver sa place dans une approche placée sous le signe des « exercices spirituels ». Le traitement du néoplatonisme est par là symptomatique de la lecture foucaldienne de l'histoire de la philosophie antique, qui se construit tout autant par ses exclusions que par ses focalisations.

SUMMARY. The interpretation of the greco-latin Antiquity developed by Michel Foucault in his last published books and his last lectures at the College de France makes little room to Neoplatonism. The only text which deals with it, L'Herméneutique du sujet, presents Neoplatonism as a simple continuation of Plato's philosophy, akin to the so-called "Platonic model" of the concern with oneself, to be then confronted by Foucault to the Hellenistic-roman and the Christian models. However, the Neoplatonic commentators of Alcibiades provided Foucault with crucial distinctions, like "political" and "cathartic" for instance, which are the common base and link of the three models that are built upon them. Furthermore, this architectonic part played by the late Neoplatonists goes along with an almost complete omission of another Neoplatonist, Plotinus, the works of which would seem at first sight far more consonant with a reading determined by the notion of "spiritual exercices". This use of 
the Neoplatonism, excluding some trends and focussing on some others, reveals Foucault's choices, and casts some light on his own style of interpreting history of ancient philosophy. 
En introduction à un ouvrage collectif portant sur le rapport de Foucault à la philosophie antique, paru il y a une dizaine d'années ${ }^{1}$, Frédéric Gros souligne à quel point l'édition des cours faits au Collège de France à partir de 1980 a élargi et transformé les perspectives que les deux derniers ouvrages publiés par Foucault, en 1984, avaient ouvertes sur la philosophie antique :

C'est à peine si l'on imaginait que l'édition de ces cours nous apprendrait quelque chose qu'on ne sache déjà : deux livres, ses derniers (L'Usage des plaisirs et Le Souci de soi) avaient en effet paru chez Gallimard en 1984, l'année même de sa mort. Ils traitaient de la manière grecque et latine de construire son rapport aux plaisirs du corps [...]. Mais voilà, il se trouve que les cours au Collège de France dépassent largement le cadre des livres publiés : les perspectives sont plus larges, l'audace spéculative plus affirmée et le cadre théorique plus ambitieux ${ }^{2}$.

Ce constat est particulièrement vaai de L'Herméneutique du sujet, précisément parce que ce cours, prenant son point de départ dans des analyses publiées dans Le Souci de soi, se présente « comme une version très élargie et amplifiée d'un seul petit chapitre du Souci de soi intitulé "e La Culture de soi" $\gg^{3}$. Le parallèle entre les deux textes fait apparaître des matériaux communs insérés dans des dispositifs interprétatifs très différents. Je me propose ici d'analyser le rôle que joue le néoplatonisme dans le dispositif propre au cours de 1982, qui est aussi le seul lieu où Foucault s'intéresse à ce courant philosophique.

Peu présent dans la réflexion que Michel Foucault conduit sur l'Antiquité, le néoplatonisme y est souvent abordé comme un simple prolongement de la philosophie de Platon. Il ne semble pas produire de nouveauté importante, qui en justifierait une analyse détaillée, contrairement au traitement appliqué à d'autres auteurs, notamment ceux du stoïcisme impérial ou de l'épicurisme. Mais il est présent, et c'est un point qui mérite d'être

1. Gros et Lévy (éd.) 2003. Les études rassemblées dans cet ouvrage interrogent le rapport de Foucault aux philosophies hellénistiques (stoïcisme, épicurisme et scepticisme) et aux dialogues de Platon.

2. Gros 2003, p. 7 .

3. Gros 2001, p. 489. 
souligné, car cette présence fait contraste avec le sort réservé à d'autres philosophes qui, eux, sont complètement absents : les sceptiques ${ }^{4}$, ou encore Aristote, qui n'est mentionné qu'à titre d'exception - « l'énigmatique exception d'Aristote $»^{5}$. La relecture que Foucault propose de l'Antiquité, en gros de Pythagore aux chrétiens, contre une lecture dominante qui s'est laissée fasciner par le seul précepte « connais-toi toi-même » sans considérer son lien constitutif et sa subordination à l'autre grande injonction antique du « souci de soi $»^{6}$, est donc une lecture fortement sélective : elle laisse de côté des courants entiers et tout à fait importants de la philosophie antique. On pourrait dire qu'elle interroge les représentations de soi à l'aide d'une pratique scénographique de l'histoire de la philosophie, et cette scénographie se construit tout autant par ses exclusions que par ses focalisations. Les usages foucaldiens du néoplatonisme - plus exactement: de différents néoplatonismes - illustrent de façon particulièrement nette ce mode de lecture qui procède par contrastes : à la fois par la place qui est faite à certains néoplatoniciens, et par celle qui est refusée à d'autres.

\section{Présence : les commentateurs néoplatoniciens tardifs}

S'intéresser à la place faite au néoplatonisme dans L'Herméneutique du sujet ne répond pas à un simple souci d'exhaustivité dans l'étude des sources que Foucault mobilise. Il s'agit plutôt de rendre visible un paradoxe que l'importance donnée à la philosophie hellénistico-romaine (je m'autorise ce raccourci auquel Foucault recourt volontiers), et plus particulièrement stoïcienne, a dans un premier temps tendance à masquer. Ce paradoxe est le suivant. Si la lecture sélective de Foucault ne juge pas utile de s'attarder en détail aux néoplatoniciens, résorbant ce courant philosophique dans la généralité de ce qu'il appelle «le modèle platonicien » du souci de soi, il n'en est que plus frappant de constater que ce sont des néoplatoniciens qui

4. Cf. Lévy 2003, p. 119-135, qui intitule son étude : « Michel Foucault et le scepticisme : réflexions sur un silence ».

5. Foucault 2001, p. 182. Cf. aussi p. 19 : « Comme chacun sait, Aristote ce n'est pas le sommet de l'Antiquité, c'en est l'exception. »

6. Cf. Foucault 2001, p. 442-443: « La tradition historique, et par conséquent la tradition philosophique [...] a toujours privilégié le gnothi seauton, la connaissance de soi, comme étant le fil directeur de toutes les analyses sur ces problèmes du sujet, de la réflexivité, de la connaissance de soi, etc. Or, à ne considérer le gnothi seauton qu'en lui-même et pour lui seul, il me semble qu'on risque d'établir une fausse continuité et d'instaurer une histoire factice, qui montrerait une sorte de développement continu de la connaissance de soi. [...] On laisse courir derrière tout cela une théorie, explicite ou implicite, mais en tout cas inélaborée, du sujet. [...] Le principe du gnothi seauton n'est pas autonome dans la pensée grecque. Et on ne peut pas, je crois, en comprendre ni la signification propre ni l'histoire si l'on ne tient pas compte de cette relation permanente entre connaissance de soi et souci de soi dans la pensée antique. » 
lui fournissent le cadre dans lequel cette lecture construit et articule les différents modèles du souci de soi - le modèle platonicien, le modèle hellénistique et le modèle chrétien ${ }^{7}$.

Ce rôle structurant apparaît clairement dans le cours du 3 février 1982, lorsque Foucault entreprend de répondre à une question de l'auditoire. Cette question touche aux principes de l'organisation générale de son travail en cours, et elle lui paraît suffisamment importante pour qu'il choisisse, pour y répondre, de modifier l'ordre prévu du cours. Il propose alors de traiter des néoplatoniciens beaucoup plus tôt qu'il n'avait pensé le faire, et de renvoyer à une séance ultérieure ce qu'il comptait aborder à ce momentlà, à savoir la parrhesia :

Justement, j'avais depuis un certain temps l'intention de me référer à deux ou trois textes tardifs mais, je crois, très éclairants sur ce problème de l'Alcibiade et sur la place qu'il occupe dans la pensée antique. Alors je vais donc faire une rocade. Au lieu de vous parler de la parrhesia maintenant et des commentateurs néoplatoniciens plus tard, je voudrais donc tout de suite évoquer un peu ce problème des commentaires néoplatoniciens de l'Alcibiade ${ }^{8}$.

La question de l'auditoire qui exige cette « rocade » porte sur le rôle séminal donné à l'Alcibiade dans le cours. Ce dialogue y est lu comme s'il précontenait toutes les directions dans lesquelles le motif du souci de soi se développera dans les siècles suivants, et Foucault emploie les termes de « germe » et de «principe » pour décrire la fonction assumée par l'Alcibiade dans l'économie générale de sa lecture. Ce rôle séminal est spécifique au cours sur L'Herméneutique du sujet, et sans commune mesure avec la place que tient l'Alcibiade dans le Souci de soi, plus précisément dans le chapitre de cet ouvrage qui a même matière que les analyses développées dans L'Herméneutique du sujet. Le parallèle entre ces deux textes offre des écarts instructifs sur la place accordée au néoplatonisme ainsi qu'à l'Alcibiade dans le cours de 1982. C'est pourquoi il me faut d'abord dire quelques mots du rapport entre ces deux textes et de la façon dont leur comparaison sera maniée ici.

Comme le rappelle Frédéric Gros en appendice à son édition du cours de 1982, Le Souci de soi, publié en 1984, donne une forme achevée et publique au cours donné en 1980-1981, soit le cours qui précède celui sur « L'Herméneutique du sujet ». Le cours de 1980-1981, consacré au thème «Subjectivité et vérité », se concentrait sur l'expérience des plaisirs dans l'Antiquité gréco-latine, en particulier dans les deux premiers siècles après

7. Pour une caractérisation globale de ces trois modèles, $c f$. Foucault 2001, p. 244-248.

8. Foucault 2001, p. 163-164. 
J.-C. L'analyse du rapport aux aphrodisia développée dans le cours de 19801981

trouvera son inscription définitive dans le Souci de soi, troisième volume de l'Histoire de la sexualité, publié en 1984. Or le cours de 1982 prend exactement comme point d'ancrage la même période historique que le cours de l'année précédente, mais avec, comme nouveau cadre théorique, celui des pratiques de soi ${ }^{9}$.

L'Herméneutique du sujet se veut donc l'exploration approfondie, selon la forme souple et libre d'un cours et dans un cadre théorique nouveau, de données présentées dans le second chapitre du tome III de l'Histoire de la sexualité $^{10}$. Autrement dit, même si Le Souci de soi est publié deux ans après la tenue du cours sur «L'Herméneutique du sujet », néanmoins, selon la temporalité de l'articulation d'un cours à un livre, qui n'est pas exactement superposable à la succession calendaire, on peut dire que Le Souci de soi représente un stade de réflexion antérieur à celui que déploie L'Herméneutique du sujet. C'est en prenant en compte cette antériorité théorique que le parallèle entre les deux textes sera utilisé ici.

Leur comparaison fait apparaître un élément important. Si le chapitre du Souci de soi portant sur «La culture de soi » développe beaucoup d'éléments qui sont repris et amplifiés dans L'Herméneutique du sujet, en revanche il n'y est quasiment pas question de l'Alcibiade. Et lorsqu'il en est question, Foucault se contente de noter que l'expression epimeleia heautou et le précepte « connais-toi toi-même » sont présents dans l'Alcibiade et y constituent des thèmes essentiels; mais aucune analyse n'est proposée de ce dialogue. Parce que le thème plus tardif de la «culture de soi » s'est constitué en « débordant son cadre d'origine et se détachant de ses significations philosophiques premières $\gg{ }^{11}$, le point de départ qu'il trouve dans l'epimeleia heautou de l'Alcibiade ne mérite pas d'être creusé. Quant au « principe delphique », « il ne serait pas suffisant d'y reconnaittre la pure et simple influence du thème socratique $\gg^{12}$, si bien que, concernant la connaissance de soi, là non plus l'étude du texte platonicien n'est d'aucune utilité. Autrement dit, souci de soi et connaissance de soi ont pris un sens nouveau et spécifique aux Ir $\mathrm{I}^{\mathrm{er}}$ et $\mathrm{II}^{\mathrm{c}}$ siècles (ce sur quoi L'Herméneutique du sujet est tout à fait d'accord), mais dans Le Souci de soi Foucault en tire la conclusion que partir de la détermination platonicienne de ce thème dans l'Alcibiade est au mieux inutile, au pire dangereux : ces points de départ

9. Gros 2001, p. 489.

10. Foucault 1984b, p. 51-85.

11. Cf. ibid.p. 59.

12. Cf. ibid.p. 74. 
risqueraient de masquer le sens spécifique qui en émerge dans la culture de soi qui se constitue à l'époque impériale.

Cette conclusion permet de mesurer nettement quelle distance sépare la démarche suivie dans L'Herméneutique du sujet de celle qui prévaut encore dans Le Souci de soi, puisque dans le cours de 1982, au contraire, l'Alcibiade tient une place majeure et fait l'objet d'une étude attentive :

Dans cet Alcibiade, posant donc le principe « connais-toi toi-même », on voit le germe de la grande différenciation qu'il doit y avoir entre l'élément du politique [...] et puis, d'un autre côté, le « connais-toi toi-même » qui appelle à un certain nombre d'opérations par lesquelles le sujet doit se purifier lui-même [...]. L'Alcibiade est donc au principe de cette fourche ${ }^{13}$.

Le changement du statut du dialogue est flagrant : désormais, Foucault considère que comprendre le souci de soi et les différentes formes qu'il a assumées à travers toute l'Antiquité doit commencer par une analyse du souci de soi tel qu'il apparaît dans ce dialogue platonicien. Cette analyse a pour objectif de déplier toutes les composantes qui sont enchevêtrées dans ce dialogue. Car c'est dans la dissociation ultérieure de ces diverses composantes que Foucault pense trouver un principe d'intelligibilité permettant de comprendre l'apparition d'autres formes du souci de soi, notamment celle qui l'intéresse le plus, à savoir le souci de soi hellénistico-romain. Ce n'est donc que dans un second temps, et sur la base d'une analyse soigneuse du souci de soi tel que le décrit Platon dans l'Alcibiade, qu'il sera possible d'identifier avec précision les traits par lesquels ce souci de soi hellénisticoromain se démarque du souci de soi platonicien, promu par contraste à la validité d'un des grands modèles du souci de soi (le «modèle platonicien $\gg{ }^{14}$ ).

Dans le cours du 3 février 1982, la question du public porte précisément sur cette nouvelle focalisation de l'analyse archéologique sur l'Alcibiade. Pourquoi choisir l'Alcibiade «pour mettre en perspective tout un pan de la philosophie ancienne $\gg{ }^{15}$ ? À cette question, Foucault répond très clairement que le rôle séminal qu'il accorde à ce dialogue lui a été désigné par les commentateurs néoplatoniciens du dialogue. Il a choisi de partir de l'Alcibiade et d'approfondir longuement ce point de départ après avoir constaté que Proclus et Olympiodore

s'accordent l'un et l'autre à considérer que ce dialogue doit effectivement être placé en tête des œuvres de Platon, que c'est par lui que l'on doit abor-

13. Foucault 2001, p. 166-167.

14. Ce modèle est décrit à plusieurs reprises : cf. Foucault 2001, p. 75, 167, 201, 244, 400.

15. Foucault 2001, p. 163. 
der l'étude de Platon ou du platonisme, et par là même l'étude de la philosophie en général. Si vous voulez, trois grands principes en effet permettent à Proclus et à Olympiodore de donner à l'Alcibiade cette place première, cette place initiale, de le mettre en quelque sorte en propylée de la philosophie $^{16}$.

Les développements que Foucault consacre aux commentaires de Proclus et d'Olympiodore, à partir d'un article de Festugière sur « L'ordre de lecture des dialogues de Platon » chez les néoplatoniciens des $\mathrm{V}^{\mathrm{e}}$ et $\mathrm{VI}^{\mathrm{e}}$ siècles ${ }^{17}$, montrent que ces textes néoplatoniciens, à ses yeux, n'ont pas seulement un intérêt au regard de la constitution d'une tradition qui ne serait que platonicienne, mais qu'ils sont aussi porteurs de la façon dont luimême trouve dans l'Alcibiade une matrice pour l'ensemble de ses analyses, en y découvrant la double préoccupation du souci de soi et du souci des autres, en y repérant le lien du souci de soi et de la connaissance de soi, bref en y trouvant tout un matériel à partir duquel le dégagement du modèle hellénistico-romain va s'opérer : par extension de l'impératif du souci de soi à la vie entière, par renversement de la subordination initiale du souci de soi au souci des autres, etc. C'est là quelque chose que signale déjà le fait que ce cours du 3 février est le seul passage de L'Herméneutique du sujet où l'on trouve, non pas des références aux néoplatoniciens en général, mais un commentaire détaillé, citations à l'appui, des analyses de Proclus et d'Olympiodore, produites dans leurs textes les plus significatifs déjà relevés par l'article de Festugière.

Cette reprise attentive des commentaires néoplatoniciens insiste sur les éléments suivants. Quels sont les « trois grands principes » en vertu desquels les néoplatoniciens ont été conduits à faire de l'Alcibiade le «propylée de la philosophie » ? Le premier est que ce dialogue est un condensé de l'ensemble de la philosophie de Platon. Le second, qu'il constitue « l'introduction, première et solennelle dans la philosophie, du gnothi seauton comme condition première de la pratique philosophique $\gg^{18}$. Le troisième, que ces commentateurs néoplatoniciens «y voient la première apparition de l'embranchement entre le politique et le cathartique ${ }^{19}$. Ayant énoncé ces trois principes, Foucault se propose aussitôt de revenir sur chacun d'eux.

Le premier principe ne semble pas avoir beaucoup d'intérêt à ses yeux, sans doute parce qu'il ne concerne que l'histoire de la philosophie platonicienne, que Proclus trouve tout entière dans l'Alcibiade, distribuée selon les catégories plus tardives de la logique, de la morale, de la physique et de la

16. Ibid., p. 164.

17. Festugière 1971 ; cité dans Foucault 2001, p. 164.

18. Foucault 2001, p. 164.

19. Ibid. 
théologie. Le second reçoit déjà plus d'attention : en mettant au principe de la pratique philosophique la connaissance de soi, l'Alcibiade des commentateurs néoplatoniciens dessine le trait majeur $\mathrm{du}$ «modèle platonicien » tel que Foucault le délimite dans L'Herméneutique du sujet, caractérisé par « l'absorption du souci de soi-même dans la forme de la connaissance de soi $\gg^{20}$. Enfin, le troisième principe du classement de tête de l'Alcibiade lui paraît encore plus intéressant, et c'est sur lui qu'il s'attarde le plus longuement. Il s'agit du repérage néoplatonicien, dans l'Alcibiade, du croisement entre deux axes promis à une séparation progressive, l'axe que Foucault appelle «cathartique » et l'axe « politique ». Olympiodore montre en effet que, dans l'Alcibiade, Platon effectue deux choix philosophiques décisifs : l'identification du soi à l'âme raisonnable, et l'inscription de cette identité dans un projet politique. Le but du dialogue est de se connaître comme âme, non comme n'importe quelle âme mais comme âme raisonnable, et non en tant que nous devons agir « de manière cathartique, ou théorique, ou théologique, ou théurgique, mais de manière politique $\gg^{21}$. De là, Olympiodore tire deux exigences pour la vie humaine, et donc deux programmes pour la philosophie: développer les vertus cathartiques et développer les vertus politiques. De ces deux programmes platoniciens, l'un serait mis en œuvre dans le Phédon, l'autre, dans le Gorgias.

Foucault fait grand usage de la distinction entre cathartique et politique dans l'économie générale de L'Herméneutique du sujet. Cet usage est double. Premièrement, la distinction entre cathartique et politique sert à démarquer les néoplatoniciens de Platon. Si Olympiodore détecte, dans l'Alcibiade, un mouvement de différenciation entre deux pratiques de soi (une pratique de soi cathartique, tournée vers soi, et une pratique de soi politique, tournée vers le gouvernement d'autrui) que les dialogues platoniciens ultérieurs développeraient de façon séparée, Foucault fait remarquer que cette différenciation doit être comprise comme une projection néoplatonicienne sur le texte platonicien, qui ne se propose nullement de dissocier ces deux axes, ni dans l'Alcibiade ni dans les autres dialogues, où la connaissance de soi et la question politique du gouvernement d'autrui dans la cité sont toujours entrelacées ${ }^{22}$. Pareille dissociation prétendument platonicienne est caractéristique, en fait, des néoplatoniciens, puisque ce sont eux qui tiennent pour problématique le lien entre « cathartique » et « politique » et qui, du coup, croient voir, déjà à l'œuvre chez Platon, cette dissociation, notamment dans la distribution thématique des différents dialogues. Tel est donc le premier usage du couple cathartique/politique

20. Ibid., p. 167.

21. Olympiodore, In Alc. 177, 3 sqq., trad. Festugière 1971, p. 541 ; cité dans Foucault 2001, p. 166.

22. Cf. Foucault 2001, p. 167-170. 
dans L'Herméneutique du sujet : dégager la spécificité du néoplatonisme par rapport à Platon lui-même, ce qui est aussi bien une manière de rendre Platon à lui-même.

Mais cette fonction n'est pas la seule, et elle n'est pas la plus importante. Le second usage de la distinction néoplatonicienne est beaucoup plus fondamental et structure le cours dans sa totalité : la distinction du politique et du cathartique sert à construire la scénographie de l'ensemble du mouvement qui, au-delà de Platon et en dehors du « modèle platonicien », conduit au thème de «l'autofinalisation » du souci de soi, thème qui anime l'émergence et la mise en place d'une culture de soi dans le modèle hellénistico-romain du sujet ${ }^{23}$. Autrement dit, si la dissociation du politique et du cathartique n'est pas pertinente pour lire Platon, contrairement à ce qu'ont cru Olympiodore et Proclus, en revanche, pour l'époque qui retient le plus l'attention de Foucault, celle des $\mathrm{I}^{\mathrm{er}}-\mathrm{II}^{\mathrm{e}}$ siècles, elle acquiert une forte valeur interprétative. Dans le modèle hellénisticoromain, «cette dissociation est déjà très largement faite » et constitue « vraisemblablement un des phénomènes les plus importants dans l'histoire de la pratique de soi, et peut-être dans l'histoire de la culture antique $»^{24}$. Car c'est en raison de cette dissociation que l'on voit le soi «se dégager peu à peu comme une fin qui se suffit à elle-même, sans que le souci des autres constitue la fin ultime et l'index qui permet de valoriser le souci de soi $»^{25}$. Si bien que, de façon assez inattendue je crois, le repérage néoplatonicien d'une part du thème cathartique (comme reformulation de l'exigence platonicienne de se connaître soi-même), d'autre part de la dissociation de ce thème par rapport au problème politique du gouvernement d'autrui, qui avait commencé par susciter l'exigence de se connaître et de se soucier de soi, et qui finit par n'être qu'un effet secondaire du souci de soi ce double repérage attribué aux néoplatoniciens est intégralement repris par Foucault lui-même pour constituer le modèle hellénistico-romain autour d'un resserrement du souci de soi sur le soi lui-même, qui cesse d'être objet d'attention de manière instrumentale par rapport à la question du politique, comme c'était le cas chez Platon.

Voici comment est introduite la spécificité de ce modèle par rapport au souci de soi platonicien :

23. Cf. p. ex. Foucault 2001, p. 170-171 : « Le soi est le but définitif et unique du souci de soi. [...] Dans le souci de soi on est son propre objet, on est sa propre fin. Il y a, si vous voulez, à la fois une absolutisation (pardonnez le mot) de soi comme objet du souci, et une auto-finalisation de soi par soi dans la pratique qu'on appelle le souci de soi. »

24. Foucault 2001, p. 170.

25. Ibid. 
C'est un phénomène important que ce détachement de ce qu'encore une fois les néoplatoniciens appelaient le cathartique, par rapport à ce qu'ils appelaient le politique. C'est un phénomène important pour deux ou trois raisons.

La première, ce serait celle-ci : pour la philosophie elle-même, le phénomène est important. Il faut bien se rappeler en effet que, depuis au moins les cyniques - les post-socratiques : les cyniques, les épicuriens, les stoïciens, etc. -, la philosophie avait de plus en plus cherché sa définition, son centre de gravité, fixé son objectif autour de quelque chose qui s'appelait la techne tou biou, c'est-à-dire : l'art, la procédure réfléchie d'existence, la technique de vie. [...] Cette question («Comment faire pour vivre comme il convient ? ») va devenir de plus en plus identique à la question, ou va être de plus en plus nettement absorbée par la question : «Comment faire pour que le soi devienne et demeure ce qu'il doit être ? » Ce qui, évidemment, va entraîner un certain nombre de conséquences. D'abord bien sûr l'absorption, de plus en plus marquée au cours de l'époque hellénistique et romaine, de la philosophie, comme pensée de la vérité, dans la spiritualité, comme transformation du mode d'être du sujet par lui-même. Avec, bien sûr, la croissance du thème cathartique. Ou encore, si vous voulez : l'apparition ou le développement de ce problème dont je vous parlerai aujourd'hui et la prochaine fois, qui est le problème fondamental de la conversion (de la metanoia $)^{26}$.

Trois mouvements fondamentaux aux yeux de Foucault - l'inflexion de la question éthique et politique du meilleur genre de vie vers la question de l'accès au vrai soi, la « croissance du thème cathartique », enfin l'apparition de la problématique de la conversion - sont pensés comme des conséquences de la dissociation entre cathartique et politique. Cette dissociation n'est pas propre au néoplatonisme, puisqu'elle lui paraît déjà opérante à l'époque du Haut-Empire, mais elle a trouvé sa formulation la plus nette dans le néoplatonisme. C'est cela qui vaut à ce néoplatonisme - tel que le conçoit Foucault, c'est-à-dire caractérisé par la conscience qu'entre cathartique et politique il y a séparation - de fournir, par une sorte de rétroprojection, une grille de lecture adéquate pour des philosophies qu'on aurait plutôt tendance à considérer d'abord comme très éloignées du néoplatonisme. Foucault dit ainsi un peu plus loin que la philosophie non chrétienne de cette époque des $\mathrm{I}^{\mathrm{er}}-\mathrm{II}^{\mathrm{e}}$ siècles de notre ère, « à la suite de ce mouvement » de séparation entre souci de soi et souci des autres, est « entièrement dominée par le thème de la cathartique ${ }^{27}$.

Faut-il le souligner ? Il est assez inattendu de voir le stoïcisme impérial ou l'épicurisme caractérisés comme des philosophies « entièrement dominées » par une exigence de katharsis, même en donnant à ce terme un sens

26. Ibid. p. 171-172.

27. Ibid. p. 172. 
un peu vague. Le thème stoïcien le plus proche de l'idée de « purification » serait probablement celui de la «discrimination » entre bonnes et mauvaises représentations, thème en effet important dans le stoïcisme impérial, mais il se dirait mieux dans le vocabulaire cognitif de la diakrisis que dans celui de la katharsis ${ }^{28}$. Même chose pour les épicuriens ou les cyniques: opérer une «purification », excepté en un sens cognitif bien précis qui ne trouverait pas sa meilleure expression dans le terme de katharsis, ne parait pas être le trait dominant de ces philosophies. Penser le souci de soi romain comme «entièrement dominé par le thème de la cathartique » ne consonne pas immédiatement avec l'image qu'on peut en avoir. Le terme même de « cathartique » est d'ailleurs présenté par Foucault comme propre au néoplatonisme ${ }^{29}$. Or, il faut souligner que cette manière de considérer le moment hellénistico-romain sous la domination d'un modèle cathartique isolé par les néoplatoniciens est une idée qui, d'abord, n'est pas non plus venue à l'esprit de Foucault. Il est intéressant, ici, de comparer à nouveau le chapitre 2 du Souci de soi à L'Herméneutique du sujet.

Dans le chapitre sur « La culture de soi », Foucault se propose de réfléchir sur la morale austère qui se développe chez les auteurs non chrétiens des deux premiers siècles de notre ère, en relation particulièrement avec les aphrodisia - morale austère qui sera reprise abondamment par les auteurs chrétiens, mais selon de tout autres intentions. Foucault insiste sur le fait que cette sévérité morale ne relève pas, dans les pratiques du Haut-Empire, de prohibitions plus nombreuses ou plus rigoureuses qu'aurait édictées le pouvoir en place (de ce point de vue, le Platon des Lois est encore plus sévère, fait-il remarquer ${ }^{30}$ ), mais plutôt d'une inflexion nouvelle du rapport de soi à soi. C'est cette inflexion nouvelle qu'il s'emploie à décrire dans tout le chapitre, par des analyses qui évoquent largement celles de L'Herméneutique du sujet :

28. Chez Épictète, un seul passage (Entretiens, IV 11, 8-16) utilise les termes $\kappa \dot{\alpha} \theta$ apoıs et $\dot{\alpha} \kappa \alpha \theta \alpha p \sigma i \alpha$. Il faut noter qu'ils y ont un sens principalement littéral, puisqu'il est question de la propreté corporelle (9-16) : pour être un homme et non un pourceau, il faut se moucher,

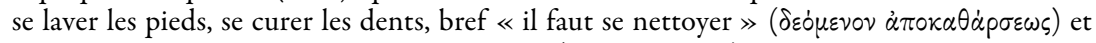
ne pas imposer aux autres sa «malpropreté » ( $\dot{\alpha} \kappa \alpha \theta \alpha \rho \sigma i \alpha, 16)$. Ce n'est que pour introduire au thème hygiénico-social de la toilette corporelle qu'Épictète en mentionne un analogue

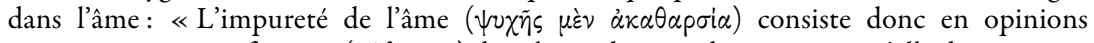
mauvaises, et sa purification ( $\kappa \dot{\alpha} \theta \alpha \rho \sigma \varsigma)$ dans la production des opinions qu'elle doit avoir. » Quant à Marc-Aurèle, on ne relève aucune occurrence de ces termes dans les Pensées.

29. Cf. Foucault 2001, p. 169 : «C'est en s'occupant d'elle-même, en pratiquant la "cathartique de soi" (terme non platonicien mais néoplatonicien) que l'âme découvre à la fois et ce qu'elle est et ce qu'elle sait »; ou encore p. 185: « Est-ce que, encore une fois pour employer le vocabulaire néoplatonicien, le politique et le cathartique sont définitivement dissociés ? »

30. Foucault 1984b, p. 55. 
Cette majoration de l'austérité sexuelle dans la réflexion morale ne prend pas la forme d'un resserrement du code qui définit les actes prohibés, mais celle d'une intensification du rapport à soi par lequel on se constitue comme sujet de ses actes ${ }^{31}$.

L'Herméneutique du sujet s'attache également à distinguer le contrôle des comportements dans le souci de soi hellénistico-romain de quelque chose qui peut y ressembler, extérieurement, mais qui en diffère foncièrement, à savoir la soumission des comportements à des règles ou à des lois :

Ce qui caractérise l'ascèse (askesis) dans le monde grec, hellénistique et romain, quels que soient d'ailleurs les effets d'austérité, de renoncement, d'interdiction, de prescription tatillonne et austère que cette askesis peut induire : elle n'est pas et n'est jamais fondamentalement l'effet d'une obéissance à la $\operatorname{loi}^{32}$.

Le chapitre sur « La culture de soi » développe alors, de façon moins étendue, les grands thèmes de L'Herméneutique du sujet : l'assujettissement de la techne tou biou au précepte ou à la forme de l'epimeleia heautou; l'extension du souci de soi à la vie entière, au-delà de la seule préparation à la vie d'adulte; l'incorporation du souci de soi dans une multiplicité de pratiques, de techniques, d'exercices («s'occuper de soi n'est pas une sinécure $\gg$ à cette époque, prévient Foucault ${ }^{33}$ ); la dimension sociale, dans l'articulation du souci de soi au soin qu'on prend du souci que les autres doivent avoir d'eux-mêmes, ce qui fait du souci de soi « une intensification des relations sociales ${ }^{34}$; la « montée du souci médical »dans cette culture de soi, avec les thèmes de la maladie, de ses progrès, de ses symptômes, ainsi que de la médication, de la cure, du secours; enfin, l'inflexion du précepte delphique, initialement introduit par le Socrate de l'Alcibiade et de l'Apologie, vers « un art de la connaissance de soi [...] avec des recettes précises, avec des formes spécifiées d'examen et des exercices codifiés $\gg^{35}$, que Foucault détaille ainsi : les procédures d'épreuve, tels les exercices d'abstinence ponctuelle; l'examen de conscience, dans sa double dimension, matinale et vespérale ; le filtrage des représentations; l'équipement de soi, comme armement et assimilation de logoi qui permettent de faire face à toutes les situations. Comme dans le cours de 1982, le rapport à soi hellénistico-romain est décrit comme l'effet d'une inflexion de la connaissance de soi vers une techne soigneusement codifiée, visant à « monter un mécanisme d'assurance » de soi contre tous les facteurs de déstabilisation,

31. Ibid.

32. Foucault 2001, p. 303.

33. Foucault 1984b, p. 66.

34. Ibid. p. 69.

35. Ibid. p. 74 
qu'il s'agisse d'une perturbatio réelle ou même seulement possible, « ce que les Grecs appellent la paraskeue $\gg^{36}$. Et ce rapport à soi, enfin, tout comme dans L'Herméneutique du Sujet, est compris à l'intérieur du « principe tout à fait général de la conversion à soi - de l'epistrophe eis heauton $»^{37}$, qui, si la formule a une «allure platonicienne ${ }^{38}$, recouvre en fait bien autre chose et vise un rapport à soi qui est de l'ordre de la possession et de la satisfaction : il s'agit de se tourner vers soi-même pour se posséder pleinement soi-même et jouir de soi, dans une tranquillité et une présence à soi « sauvées », mises à l'abri des aléas de la fortune. Bref, le phénomène analysé est exactement le même que dans L'Herméneutique du sujet, selon les mêmes lignes de force, les mêmes concepts - sauf un : on remarquera que, dans cette première mise en place des principaux éléments sur lesquels L'Herméneutique du sujet s'attarde plus longuement, il n'est jamais question de «purification ». La diakrisis des représentations est évoquée, avec insistance, le détournement de l'agitation extérieure et le retournement vers soi, mais rien de tout cela n'appelle à être dit ni pensé selon un modèle « cathartique ». Par ailleurs la mention de l'Alcibiade, comme je l'ai déjà signalé, est peu appuyée, et il n'est pas question des néoplatoniciens. Seuls deux médio-platoniciens sont cités, Albinus et Apulée ${ }^{39}$, dans un contexte où il s'agit de témoigner de l'omniprésence du thème de l'epimeleia heautou dans des écoles philosophiques très diverses: leur nom est simplement ajouté, dans une liste qui vaut par son éclectisme, à ceux d'Épicure, Sénèque, Musonius, Marc-Aurèle, Épictète, Pline, etc. Pourtant le texte d'Albinus qui est alors cité porte précisément sur la nécessité de commencer l'étude de la philosophie par la lecture de l'Alcibiade, et cette citation est extraite de l'article de Festugière dont Foucault parle dans L'Herméneutique du sujet ${ }^{40}$ - mais, dans le chapitre 2 du Souci de soi, il n'en tire rien. Il se contente d'énumérer là un témoignage, parmi tant d'autres, de la pénétration de l'epimeleia heautou ou de la cura sui dans les cercles philosophiques les plus variés.

Le chapitre suivant éclaire cette inattention à quelque chose qui joue au contraire dans L'Herméneutique du sujet un rôle structurant. C'est que, se proposant de rechercher « dans quel contexte et pour quelles raisons la culture de soi s'est ainsi développée, et précisément sous la forme qu'on vient de voir $\gg^{41}$, Foucault a en tête un tout autre schéma d'intelligibilité que

36. Foucault 2001, p. 91.

37. Foucault 1984 b, p. 81 . Sur la conversion à soi, $c f$. le cours du 10 février $1982,1^{\text {re }}$ heure (Foucault 2001, p. 197 sqq.).

38. Ibid.

39. Foucault 1984 b, p. 59.

40. Cf. ibid. n. 1, qui renvoie à Festugière 1971, p. 536.

41. Foucault 1984b, p. 85. 
celui que lui fournissent Olympiodore et Proclus dans L'Herméneutique du sujet. Dans le Souci de soi, il a en tête un schéma qu'on pourrait dire sociopolitique ou socio-culturel, et non cette espèce d'interprétation de la philosophie par elle-même qu'il s'attache à ausculter dans le cours de 1982, en tant qu'elle est constitutive d'une certaine histoire du sujet. L'explication socio-politique est développée au chapitre suivant du tome III de l'Histoire de la sexualité, intitulé «Soi et les autres $»^{42}$. Foucault s'appuie sur «les travaux des historiens », qui « peuvent suggérer plusieurs motifs » au développement de la culture de soi et à l'inflexion corrélative de l'évaluation des plaisirs vers une morale plus austère. Il isole deux de ces motifs comme particulièrement importants : « des changements dans la pratique matrimoniale et des modifications dans les règles du jeu politique $\gg^{43}$. Deux points que développe le chapitre 3 du Souci de soi, faisant de la culture de soi « une réponse originale, sous la forme d'une nouvelle stylistique de l'existence » à ces deux « modifications sociales ${ }^{44}$. Peu importe ici comment Foucault comprend cette réponse. Plus intéressante est la différence du registre explicatif proposé, ici et là, pour un même phénomène. Car, bien entendu, dans L'Herméneutique du sujet il n'est pas du tout question, pour rendre compte de la spécificité du souci de soi hellénisticoromain, d'invoquer l'évolution du mariage ou le nouveau jeu politique. Le phénomène analysé dans L'Herméneutique du sujet comme «autofinalisation du souci de soi » est bien le même que celui décrit comme « culture de soi » dans le tome III de l'Histoire de la sexualité, mais l'explication en est tout autre, et c'est ce changement de registre explicatif qui conduit à l'étrange manière de penser le rapport à soi dans le modèle hellénistico-romain comme exemplaire de l'axe «cathartique » clairement dégagé et formulé grâce aux néoplatoniciens. Sur ce point, il faut donner toute son importance, je crois, à la façon déjà signalée dont, dans L'Herméneutique du sujet, Foucault en vient à parler des néoplatoniciens : suite à la question du public sur l'importance nouvelle donnée à l'Alcibiade, il choisit de bousculer l'ordre initial de son cours, et, abandonnant un parcours chronologique, fera une « rocade » et parlera d'abord des néoplatoniciens, et ensuite seulement du reste. Je crois que cette disposition a son importance : les néoplatoniciens sont certes postérieurs aux philosophes des $\mathrm{I}^{\mathrm{er}}-\mathrm{II}^{\mathrm{e}}$ siècles, mais si l'on suit l'ordre méthodologique des principes et des repères que se donne l'herméneutique foucaldienne - cet ordre de la construction et de l'opposition de grands pôles ou grands schèmes de pen-

42. Chapitre 3: «Soi et les autres », dans Foucault 1984b, p. 89-117 (I : « Le rôle matrimonial »; II : « Le jeu politique »).

43. Foucault 1984 b, p. 89 .

44. Ibid. 
sée : les «modèles 》 du souci de $\mathrm{soi}^{45}$-, alors il faut commencer par les néoplatoniciens, parce que leur grande distinction entre vertus politiques et vertus cathartiques doit être placée au point de départ de la constitution du modèle romain du souci de soi, caractérisé entre autres par la secondarité de l'axe politique, et donc par la prééminence du rapport de soi à soi sur le rapport de soi à autrui.

Si la présence du néoplatonisme des commentateurs tardifs est circonscrite à quelques séances du cours de 1982 et reste par là relativement discrète, c'est donc par une discrétion que l'on comparerait volontiers à celle des éclairagistes dans un spectacle. Placés en retrait, invisibles ou presque, ce sont eux cependant qui donnent du relief ou au contraire laissent dans l'ombre les acteurs sur la scène. À ce titre, ils sont les opérateurs fondamentaux de toute scénographie.

\begin{abstract}
Absence : Plotin
Un des effets interprétatifs de la façon dont Foucault mobilise la distinction néoplatonicienne du cathartique et du politique est que, sous un certain point de vue, le néoplatonisme obéit au même mouvement que la philosophie hellénistique et romaine. Ces deux courants de la pensée ancienne partagent ce grand trait qui consiste à rompre l'alliance, «l'arrimage » entre souci de soi et souci des autres dans le platonisme ${ }^{46}$, et à renverser la subordination du premier au second, en faisant du souci politique ou souci d'autrui une sorte d'effet, non recherché pour lui-même et finalement inessentiel, du souci de $\operatorname{soi}^{47}$. Cependant, dans le pointage auquel se livre Foucault des diverses figures philosophiques sur la carte des voies ouvertes par l'Alcibiade, cela est sans conséquence philosophique sur le statut du néoplatonisme lui-même: le néoplatonisme n'en reste pas moins inclus dans le modèle platonicien du souci de soi. Tout se passe comme si Foucault utilisait une distinction fondamentale que lui fournit le néoplatonisme, qui devrait s'appliquer en premier lieu au néoplatonisme

45. Sur cette pratique de l'opposition des concepts réinsérée dans une distinction des moments historiques, $c f$. Jaffro 2003, p. 78-79: « Foucault ne procédait ni par argumentation ni par construction de concepts [comme le faisait Deleuze], mais selon une méthode différentielle qui lui était propre: l'opposition de concepts et la dramatisation de cette opposition au moyen d'une représentation historique sous la forme d'une distinction d'époques. Si l'on veut, la méthode de Foucault n'était pas la raison dans l'histoire, mais l'histoire dans la raison. C'est pour cela que l'histoire alternative du sujet nécessitait le plus grand dépaysement possible et la plongée la plus "archéologique", afin d'avoir pour effet la plus grande transformation possible, et qu'elle ne pouvait pas alors ne pas s'accompagner d'importantes distorsions. » 170.

46. Sur cet «arrimage », assuré de triple façon par Platon, cf. Foucault 2001, p. 169-

47. Cf. notamment Foucault 2001, p. 170, 198, 429.
\end{abstract}


lui-même, pour penser tout autre chose que lui, et sans s'interroger sur les éventuelles transformations induites, chez les néoplatoniciens eux-mêmes, par la domination non platonicienne du souci de soi sur le souci des autres. C'est pourquoi, en dehors de l'attention méthodologique à la dissociation entre cathartique et politique, pour le reste, dans L'Herméneutique du sujet, le néoplatonisme semble bien n'avoir d'autre importance que celle d'un avatar du platonisme, et l'exploration des mutations et de l'expansion du souci de soi dans l'Antiquité peut le laisser de côté. Foucault ne trouve pas chez Plotin, par exemple, autre chose que chez Platon: il s'intègre au « modèle platonicien », il le prolonge, et tout est dit. Pourtant, le plotinisme semble être, à côté des commentateurs de l'Alcibiade, un autre aspect du néoplatonisme que Foucault connaît. À la lettre, Plotin ne fait pas partie des absents du cours de 1982 : son nom y est cité, certes avec parcimonie (deux fois seulement me semble-t-il), dont une citation assortie de la référence à un traitét ${ }^{48}$. Cependant, malgré ces quelques citations, qui autorisent à penser que Foucault lisait Plotin même s'il en parle très peu, les références au néoplatonisme, en dehors de l'usage des commentaires sur l'Alcibiade dont j'ai déjà parlé, sont des références générales, qui servent à confirmer des constantes, non à pointer des discontinuités.

Cet usage du néoplatonisme en forme de confirmation est d'abord appliqué à des traits communs à la pensée antique tout entière, dont le repérage par Foucault se marque souvent par des listes. Par exemple, la liaison constante, dans toutes les écoles philosophiques anciennes, entre la question de la philosophie (comment accéder à la vérité) et celle de la spiritualité (quelles transformations de l'être même du sujet sont nécessaires pour accéder à la vérité $)^{49}$ s'énonce dans une liste : «Chez les pythagoriciens, chez Platon, chez les stoïciens, les cyniques, les épicuriens, chez les néoplatoniciens, etc. $\gg^{50}$. Le second lieu d'application de cet usage du néoplatonisme sur le mode de la confirmation est interne au platonisme : la confirmation de constantes à l'intérieur du « modèle platonicien », ou encore l'identification des persistances de ce modèle au-delà de Platon, non

48. Cf. Foucault 2001, p. 11, 199.

49. Sur l'articulation de ces deux questions, inséparables dans toute l'Antiquité tant païenne que chrétienne, $c f$. entre autres passages Foucault 2001, p. 16-20. Leur séparation, préparée par la théologie et réalisée dans le «moment cartésien », marque le passage à « un autre âge de l'histoire des rapports entre la subjectivité et la vérité » (p. 20, cf. aussi la variante manuscrite signalée en note p. 172, et la discussion p. 183-184).

50. Foucault 2001, p. 18. Autre exemple de liste mentionnant Plotin, lorsqu'il est question de la récurrence du terme et des images de la conversion vers soi-même : «Vous avez par exemple cette expression - que vous trouvez chez Épictète, que vous trouvez chez Marc Aurèle, que vous trouvez aussi chez Plotin - qui est : epistrephein pros heauton (se tourner vers soi, se convertir à soi). Vous trouvez chez Sénèque une expression comme [se] convertere ad se (se convertir à soi). » (p. 199) 
plus selon la méthode de la liste et du « etc.», mais selon celle de l'évidence, du «bien sûr ». Ainsi, à propos de techniques de soi archaïques rélelaborées par Platon: «Bien sûr, les techniques de ce genre, vous les retrouvez chez les néoplatoniciens et les néopythagoriciens, ça va de soi $\gg^{51}$. Dans ce second usage, on a affaire à la constitution d'une tradition 《 platonicienne et néoplatonicienne ${ }^{52}$, d'une «longue continuité »du «platonisme classique » au néoplatonisme ${ }^{53}$, dans laquelle le moment néoplatonicien ne fait que confirmer, en les présentant sous une forme achevée, les articulations platoniciennes. On y « retrouve», «ça va de soi », « bien sûr », ce qui déjà se trouve chez Platon, tout particulièrement deux traits caractéristiques du modèle platonicien : l'importance de la connaissance de soi comme vecteur presque exclusif du souci de soi, et l'articulation entre connaissance de soi, accès au divin et reconnaissance du divin en soi. Même si le néoplatonisme est désormais soumis au régime de la dissociation entre cathartique et politique, même s'il ne borne plus le souci de soi aux préparatifs à la vie adulte mais l'étend à la vie entière, ce sont là des différences par rapport à Platon qui ne sont pas pensées comme productrices d'écarts, de discontinuités, de singularités - comme c'est le cas en revanche pour les écoles hellénistiques, et ce à partir des mêmes différences.

On peut se demander ce que signifie cette conception du néoplatonisme selon la figure de l'avatar platonicien. On a vu que, lorsque cela lui paraissait utile, Foucault n'hésitait pourtant pas à pointer des dissociations et des renversements entre Platon et sa postérité platonicienne. Cela veut-il dire qu'en dehors de l'apport structurant de Proclus ou d'Olympiodore, Foucault n'a rien trouvé dans le néoplatonisme qui lui soit utile pour scénariser l'histoire du sujet et des rapports à soi ? Je voudrais indiquer un argument qui autorise une hypothèse plus forte: que Foucault, dans le cours de 1982, met délibérément de côté un autre néoplatonisme, celui de Plotin, non parce qu'il lui paraît être une pièce inutile dans son projet, mais parce qu'il y serait un élément perturbateur.

Cet argument est le suivant. Il paraît tout de même très étrange que Foucault ne s'intéresse pas davantage à Plotin, si l'on prend en compte deux aspects, constants dans ses cours comme dans ses ouvrages, de sa manière de faire concernant l'accès aux textes antiques. Le premier aspect en est une pratique affichée de l'accès de seconde main, via des «passeurs » qui sont des ouvrages classiques de grands antiquisants (Pierre Hadot, Paul Veyne, Peter Brown, André-Jean Festugière $\left.{ }^{54}\right)$. Le second, une forme d'attention

51. Foucault 2001, p. 49 ; je souligne.

52. Par exemple Foucault 2001, p. 75.

53. Foucault 2001, p. 401.

54. Cf. notamment l'Introduction de Foucault 1984a, p. 13-14. 
aux textes antiques qui privilégie les images et les « petits concepts $»^{55}$ plutôt que les dogmata et les fondements théoriques (ainsi dans le stoïcisme : la navigation, l'athlète, le traitement médical, plutôt que la doctrine des kathekonta, la définition du telos, etc. $\left.{ }^{56}\right)$. Or, en fonction de ces deux modalités du rapport de Foucault aux textes antiques, il paraît très étonnant que Foucault fasse une place si minime et si insignifiante à Plotin.

La première cause de cet étonnement est bien sûr qu'un des «passeurs » reconnus de Foucault, Pierre Hadot, est l'auteur de travaux sur Plotin qui, menés dans la perspective des «exercices spirituels », avaient toutes les chances de retenir l'attention de Foucault. Pierre Hadot nous dit que, du propre aveu de Foucault, ce dernier « avait été un lecteur attentif de certains de $[\mathrm{s}]$ es travaux, $[. .$.$] et surtout de l'article liminaire "Exercices$ spirituels" de l'Annuaire de la $V^{e}$ section de l'École Pratique des Hautes Études pour l'année 1975-1976 $»^{57}$. Or, dans cet article, on trouve de nombreux développements sur Plotin, qui insistent sur les aspects qui intéressent justement Foucault dans la pensée antique : une pratique de la philosophie qui rend inséparable la connaissance de la vérité d'un travail sur soi et d'un souci de soi. La seconde cause d'étonnement touche à l'autre caractéristique du rapport de Foucault aux textes anciens, à savoir son attachement aux images et aux «petits concepts » plus qu'aux fondements doctrinaux. Car Plotin, et en particulier le Plotin qu'il pouvait rencontrer chez Hadot, avait, de ce point de vue-là, de quoi le séduire. Par quoi ? De toute évidence, par l'image célèbre que Pierre Hadot a mise en exergue à son petit livre Plotin ou la simplicité du regard ${ }^{58}$ et sur laquelle il s'arrête longuement dans l'article consacré aux «Exercices spirituels » : l'image de la sculpture de soi. Voici ce que Foucault a pu lire sous la plume de Pierre Hadot, et dont Pierre Hadot lui-même confirme qu'il a été un « lecteur attentif », à propos de cette image :

Une expression plotinienne symbolise bien cette finalité des exercices spirituels, cette quête de la réalisation de soi : sculpter sa propre statue. Elle est d'ailleurs souvent mal comprise, car on s'imagine facilement que cette expression correspond à une sorte d'esthétisme moral; elle signifierait: prendre une pose, choisir une attitude, se composer un personnage. En fait il n'en est rien. Pour les Anciens, en effet, la sculpture était un art qui « enlève » par opposition à la peinture qui est un art qui « ajoute » : la statue préexiste dans le bloc de marbre et il suffit d'enlever le superflu pour la faire apparaître. Cette représentation est commune à toutes les écoles

55. L'expression est de Bénatouil 2003, p. 25, qui donne comme exemple de « petits concepts » le salut, l'askesis, la paraskeue, le procheiron, la melete, la parrhesia.

56. Cf. Gros 2001, p. 502-503.

57. Cf. Hadot 1993a, p. 229. Cet article se trouve dans Hadot 1993b, p. 13-58.

58. Hadot 1963 
philosophiques: l'homme est malheureux parce qu'il est l'esclave des passions, c'est-à-dire parce qu'il désire des choses qui peuvent lui échapper, parce qu'elles lui sont extérieures, étrangères, surperflues. Le bonheur consiste donc dans l'indépendance, la liberté, l'autonomie, c'est-à-dire dans le retour à l'essentiel, à ce qui est véritablement « nous-mêmes » et à ce qui dépend de nous ${ }^{59}$.

Hadot poursuit en prenant plusieurs exemples qui confirment la transversalité, par rapport à la diversité des écoles philosophiques, de ce schème de la sculpture de soi : la métaphore platonicienne de Glaucos, le dieu marin recouvert de coquillages et d'algues, dont il faut racler les concrétions superficielles pour en retrouver la forme véritable, semblable à l'âme dont la « vraie nature apparaîtrait si elle sortait de la mer en rejetant loin d'elle tout ce qui lui est étranger $\gg{ }^{60}$; l'usage stoïcien de la distinction entre ce qui dépend de nous et ce qui n'en dépend pas, qui invite à « rejeter tout ce qui nous est étranger pour revenir à notre véritable moi $\gg{ }^{61}$; ou encore l'utilisation épicurienne d'une distinction analogue, car dotée de la même fonction, entre les désirs vains et les désirs naturels et nécessaires. L'image plotinienne de la sculpture de soi exprime, conclut Hadot, le sens proprement antique de «tout exercice spirituel [qui] est donc, fondamentalement, un retour à soi-même, qui libère le moi de l'aliénation où l'avaient entraîné les soucis, les passions, les désirs $\gg^{62}$.

Faisons un peu de fiction. Il serait très facile d'imaginer que Foucault, ayant lu cet article - et il l'a lu -, s'empare du motif plotinien de la sculpture de soi pour en faire un grand schème expressif de la fonction « éthopoétique » d'un savoir gréco-latin « capable de produire un changement dans le mode d'être du sujet ${ }^{63}$, que l'introduction à L'Usage des plaisirs décrit déjà comme une manière de «se façonner soi-même comme sujet éthique $\gg^{64}$. On s'attendrait à ce que cette image rivalise sans peine, jusqu'à les éclipser, avec les grandes images de la navigation, de la médication ou de l'athlète, que Foucault reprend souvent et qu'il fixe comme des concentrés de sens. Car c'est une image qui se prête, plus que toute autre peut-être, à

59. Hadot 1993b, p. 48-49. À ce passage on peut ajouter des déclarations générales comme : «L'importance des exercices spirituels dans la philosophie de Plotin est capitale » ou la « transfiguration de la personnalité » est l'aboutissement des « expériences de Plotin » (Hadot 1993b, p. 47). Pierre Hadot met tout naturellement en tête de son ouvrage Plotin ou la simplicité du regard, comme résumant l'esprit de la philosophie plotinienne, l'injonction de la sculpture de soi formulée en Enn. I, 6 [1] 9, 13 : « Ne cesse de sculpter ta propre statue. » (《I. Portrait », Hadot 1963, p. 9)

60. Hadot 1993b, p. 49.

61. Ibid.

62. Ibid.

63. Foucault 2001, p. 228.

64. Foucault 1984a, p. 19. 
un usage foucaldien, y compris bien entendu pour y lire autre chose que ce que Plotin y a mis, et notamment pour lire les stoïciens. Épictète par exemple, développant l'idée que c'est en observant ses propres actions, non en récitant des doctrines, que chacun peut savoir à quelle secte il appartient, recourt à cette image pour exprimer le pouvoir de façonnement que possèdent les logoi stoïciens :

Qui donc est stoïcien ? Comme nous appelons statue de l'école de Phidias

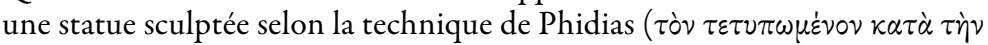

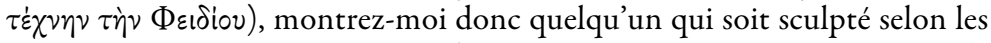
dogmes qu'il affirme en paroles ( $\kappa \alpha \tau \grave{\alpha} \tau \grave{\alpha} \delta \dot{\delta} \gamma \mu \alpha \tau \alpha \grave{\alpha} \lambda \alpha \lambda \varepsilon \tilde{\imath} \tau \varepsilon \tau U \pi \omega \mu \dot{\varepsilon} v \sigma \nu)$. Montrez-le moi malade et heureux, en danger et heureux, mourant et heureux, méprisé et heureux. Allons, montrez; par tous les dieux, je veux voir un stoïcien. Mais vous n'avez à me montrer personne qui soit entièrement

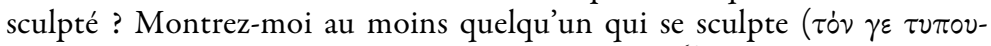
$\mu \varepsilon \dot{v} \circ v \delta \varepsilon i \xi \alpha \tau \varepsilon)$, qui ait du penchant pour le stoïcisme ${ }^{65}$.

Mais cette attente est tout à fait déçue par la lecture de L'Herméneutique du sujet: on n'y trouve pas l'image de la sculpture de soi-même. Au long des presque cinq cents pages que compte l'édition du cours de 1982, seules deux références très rapides sont faites à la statuaire, dans le cadre de l'analyse d'un point mineur au regard de l'ensemble, celui des dispositions requises pour qu'un cours de philosophie produise des effets sur ses auditeurs. Rapprochant deux textes, l'un de Sénèque, l'autre d'Épictète ${ }^{66}$, Foucault dégage deux conditions : l'auditeur, selon Sénèque, doit être concentré, exigence qui, ajoute Foucault, relève du « registre de l'immobilité de la statue ${ }^{67}$; et l'enseignement, selon Épictète, doit se réaliser à la fois

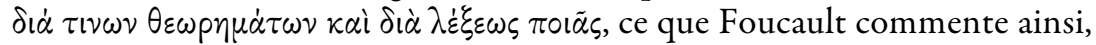
insistant sur la lexis : «pour éviter de parler de façon vaine ou de façon nuisible, il faut quelque chose qui est une techne, un art. Il faut également, pour sculpter comme il faut, une certaine techne $\gg^{68}$. Rien de plus, la chose est dite en passant.

On est donc loin de l'exploitation que l'on pourrait attendre des ressources sémantiques de la «sculpture de soi » pour décrire un mode de rapport à soi qui consiste pourtant à « faire de sa vie l'objet d'une techne, faire de sa vie par conséquent une ouvre - œuvre qui soit (comme doit l'être tout ce qui est produit par une bonne techne, une techne raisonnable)

65. Entretiens II 19, 23-25, trad. Bréhier 1962 modifiée.

66. Lettre 52, 9-12 et Entretiens II 23, 40. Aucun de ces deux textes ne recourt au vocabulaire ni à l'image de la statuaire.

67. Foucault 2001, p. 328.

68. Ibid. p. 323. 
belle et bonne $\gg^{69}$. Le silence fait sur cette image, dès lors qu'on sait que Foucault la connaissait bien au moins grâce à sa lecture de Pierre Hadot, me paraît si étonnant que j’y vois un silence volontaire, c'est-à-dire une énigme à résoudre. Je ferai donc quelques hypothèses sur les raisons d'un tel silence.

Il faut remarquer tout d'abord que la sculpture de soi est une image qui n'est pas très répandue dans les textes stö̈ciens, même ceux de la période impériale. Elle vient pourtant assez naturellement sous la plume d'un spécialiste de la philosophie hellénistique, pour résumer de façon suggestive la spécificité du rapport à soi et au monde propre au stoïcisme. Jacques Brunschwig, analysant l'attitude des stoïciens en matière d'engagement politique, conclut ainsi : «Aux lents progrès de la justice dans le monde [le stö̈cien] préfère l'admirable statue que sculptent de lui-même ses gestes, et au besoin son suicide ${ }^{70}$. » Étrangement, la formule, qui résume bien l'esprit du stoïcisme, tire peut-être sa lettre d'une projection plotinienne : car les textes des stoïciens n'utilisent que peu cette métaphore. Non seulement l'image n'y est pas courante, mais, lorsqu'on l'y trouve, elle a souvent une portée négative. Marc Aurèle ne l'utilise pas ${ }^{71}$. Épictète illustre par elle ce que l'apatheia ne doit pas être, à savoir une indifférence radicale à autrui qui ferait négliger les kathekonta: si le premier objectif d'un homme doit être de se soustraire aux troubles de l'âme, le second est qu' « il ne faut pas être impassible à la manière d'une statue $(\dot{\alpha} \pi \alpha \theta \tilde{\eta} \dot{\omega} \varsigma \dot{\alpha} \nu \delta \rho \dot{\alpha} \nu \tau \alpha)$; il faut maintenir nos rapports naturels ou acquis avec autrui, comme homme religieux, comme fils, comme frère, comme citoyen $\gg^{72}$. C'est pourquoi la statue peut aussi servir à critiquer la démonstrative conception de l'apatheia qui s'observe chez les cyniques : dire que la philosophie est un exercice ne signifie pas qu'elle réclame des exploits physiques dignes de « faiseurs de tours », comme « marcher sur une corde raide [...] ou embrasser des statues » au marbre glacé en plein cœur de l'hiver ${ }^{73}$.

69. Ibid.p. 405.

70. Brunschwig 1998, p. 560.

71. Dans les Pensées pour moi-même, on ne trouve aucune occurrence d' $\not \alpha \gamma \lambda \mu \alpha$ et une seule, insignifiante, d'å $\nu \delta$ pi $\alpha$, en I 17 : « Il est possible de vivre à la cour sans avoir besoin de gardes du corps, de vêtements de parade, de lampadaires, de statues, de choses analogues et d'un luxe semblable [...] », trad. Meunier 1933.

72. Entretiens, III 2, 4, trad. Bréhier 1962. Dans le même registre de l'insensibilité stérile, en III 9, 12 un auditeur se plaint de s'être « entretenu avec Épictète comme si c'était avec une pierre ou une statue $\gg$.

73. Entretiens, III 12, 3, faisant allusion à une anecdote dont Diogène Laërce se fait l'écho en Vies, VI 23 : «L'été, [Diogène] se roulait sur du sable brûlant, tandis que l'hiver, il étreignait des statues couvertes de neige, tirant ainsi profit de tout pour s'exercer. » (trad. Goulet-Cazé 1999) L'allusion, avec la même signification négative, se retrouve en Entretiens, IV 5, 14 et Manuel, 47 : « Si tu veux t'entraîner à la peine, fais-le pour toi-même, et non pour les étrangers; n'embrasse pas les statues. » (Trad. Bréhier 1962.) 
Sénèque est un peu plus enclin à utiliser la métaphore de la sculpture, et recourt parfois au verbe «se façonner » pour exprimer le progrès moral. Un texte en particulier le fait en explicitant la référence à la statuaire, citant Virgile: «Façonne ton être; rends-le, lui aussi, digne du dieu ${ }^{74}$. » Il s'agit dans ce passage de savoir ce qui rend un homme égal au dieu, tout au long du chemin de sa vie. Sénèque écarte un certain nombre de biens matériels qui ne sauraient nous égaler aux dieux, précisément parce que les dieux sont des dieux sans posséder ce genre de biens :

Mais ce n'est pas l'argent qui fera de toi l'égal du dieu ; le dieu n'a rien. Ni la toge de magistrat : le dieu est nu. Ni la réputation, ni toute une mise en scène autour de ta personne, la connaissance de ton nom propagée parmi les peuples : nul ne connaît le dieu ; beaucoup jugent mal de lui, et impunément $^{75}$.

La possession d'esclaves, la beauté, la force, sont aussi disqualifiées, la première parce que le dieu n'a pas besoin d'esclaves pour porter le monde tout entier, il le porte lui-même; les secondes parce qu'elles ne résisteront pas à la vieillesse. Pour devenir «par deo», il ne faut pas compter sur ce genre de possessions matérielles et éphémères, il faut chercher

quelque chose qui ne se détériore pas de jour en jour et à quoi rien ne puisse faire obstacle. Et quelle est cette chose ? C'est l'âme, j'entends une âme droite, bonne et grande. On ne saurait la nommer qu'en disant : c'est un dieu qui s'est fait l'hôte d'un corps mortel ${ }^{76}$.

Et de commenter alors le vers virgilien en ces termes : «Mais tu ne façonneras pas l'ouvrage dans l'or ou dans l'argent, matière inapte à reproduire en traits ressemblants l'image de la divinité. Songe qu'au temps où nous nous faisions écouter des dieux, ils étaient d'argile $\mathrm{e}^{77}$. $\gg$ Ce qui appelle la métaphore de la sculpture, ici, ce n'est donc pas l'activité ellemême du fingere, comme image du travail continu, soigneux, et à belle visée, sur soi-même. Dans ce texte, qui insiste plus sur la matière appropriée à la sculpture de soi que sur la technique et la gestuelle du façonnement, la métaphore statuaire a clairement une portée négative: il s'agit de disqualifier l'élévation de soi qui choisit les voies du faste, de la supériorité sociale, du nom, et la référence à la statue est d'abord une référence critique à l'élévation de statues d'or et d'argent pour célébrer des hommes (allusion peut-être à la statuaire impériale). Ce n'est que pour rejeter cette mauvaise

74. Énéide, VIII, 365.

75. Lettres à Lucilius, 31, 10, trad. Noblot 1993.

76. Ibid. 11.

77. Ibid. On trouve un texte parallèle chez Épictète, Entretiens, II 19, 26. 
divinisation de soi que Sénèque en vient à formuler l'exigence de se façonner soi-même pour se rendre égal à un dieu : son but est d'insister sur le matériau approprié, qui doit être aussi modeste que l'argile.

Bref, après avoir été déçu de ne pas trouver de sculpture de soi chez Foucault, il faut accepter d'être déçu de ne la trouver guère plus chez ses auteurs préférés, auxquels pourtant elle semblerait aller comme un gant. Mais bien sûr, cela ne suffit pas à expliquer que Foucault ne s'y intéresse pas. Après tout, il n'hésite pas à s'emparer du thème néoplatonicien de la purification pour en faire le schème dominant de l'épicurisme ou du stoïcisme impérial. La faible présence de la métaphore de la sculpture chez les stoïciens n'est pas en soi une raison susceptible d'expliquer pourquoi Foucault n'a pas ici procédé de même.

Alors, revenons à Plotin lui-même, et à son injonction : « Ne cesse pas

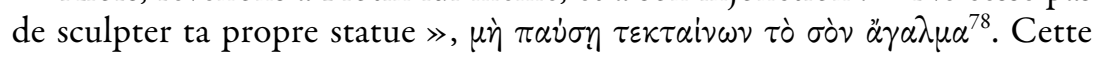
image développée dans le traité Sur le Beau part très probablement d'une citation partielle du Phèdre:

Ainsi, en ce qui concerne l'amour des beaux garçons, chacun fait le choix qui correspond à sa manière d'être, et, comme si le garçon choisi était le dieu en personne, se fabrique pour soi-même une statue à sa ressemblance

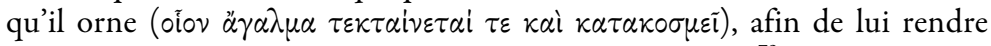
hommage et de le célébrer comme on célèbre des mystères ${ }^{79}$.

La filiation entre ce passage du Phèdre et le traité Sur le Beau est évidemment scellée par l'identité du thème : ici et là, il est question de la beauté comme de ce qui suscite l'amour et fait passer l'âme amoureuse du sensible à l'intelligible. Le texte de Plotin est d'ailleurs parsemé d'autres références au Phèdre. Les deux textes toutefois diffèrent notablement. Ils se réfèrent à deux étapes différentes de l'ascension érotique telle que la décrit Platon dans le Banquet, et que le traité Sur le Beau reprend, degré après degré ${ }^{80}:$ le passage du Phèdre concerne l'amour des beaux garçons, alors que le chapitre 9 du traité Sur le Beau en est déjà, lui, à la beauté de l'âme

78. Enn. I 6 [1] 9, 13.

79. Phèdre, 252d5-e1, trad. Mouze 2007. Le développement plotinien qui fait des gestes du sculpteur un modèle pour inciter l'âme à purifier en elle sa part la meilleure, à savoir son « œil intérieur », fait sans doute aussi écho à République, 533d2, en transposant dans le registre sculptural la métaphore - certes nettement moins poétique, mais consonante - du nettoyage (voir également République, 518c-d).

80. Enn. I 6 [1] 1, 16-20: « Qu'est-ce donc que cette beauté qui est présente dans les corps ? C'est en fait ce qu'il faut examiner en premier. Qu'est-ce donc qui meut les yeux des spectateurs, les tourne vers lui, les attire, et fait se réjouir au spectacle ? Car, trouvant cela,

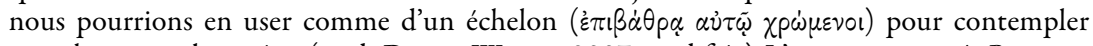
aussi les autres beautés »(trad. Darras-Worms 2007 modifiée) L'image renvoie à Banquet,

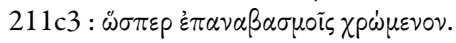


vertueuse, de la $\psi v \chi \grave{\eta} \dot{\alpha} \gamma \alpha \theta \dot{\eta}(9,6)$. Ce décalage s'illustre dans une autre différence remarquable. Dans le Phèdre la métaphore de la statue est traitée sur le mode de l'extériorité : l'amant élève une statue à son aimé, comme il le ferait pour honorer un dieu, et il orne cette statue - on est dans la métaphore de l'ajout, de l'addition ornementale, non de la suppression du superflu. Plotin intériorise doublement l'image: la statue n'est plus autre que le soi, et elle requiert des actions qui ôtent le superflu et enlèvent ce qui est étranger, non qui ajoutent à l'identité.

L'injonction de sculpter sa propre statue intervient au moment où, après que l'âme a été accoutumée à voir la beauté des $k \alpha \lambda \grave{\alpha}$ êp $p \alpha$, elle doit arriver à voir la beauté de l'âme de ceux qui font ces actes nobles. Il s'agit donc de passer du spectacle extérieur des actions admirables au spectacle de leur principe intérieur :

Mais comment pourrais-tu voir quelle est la sorte de beauté que possède

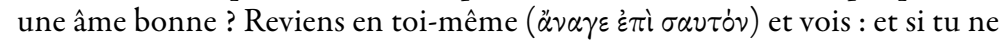
t'es pas encore vu toi-même comme beau, fais comme le sculpteur d'une

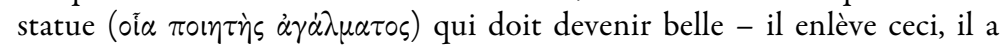

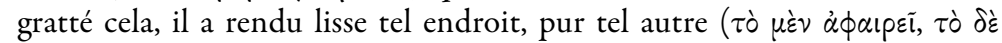

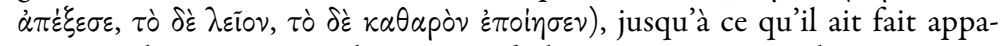
raître un beau visage sur la statue - de la même manière enlève, toi aussi,

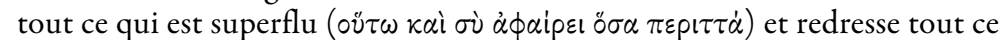
qui est tordu, purifie tout ce qui est obscur puis travaille à le rendre brillant,

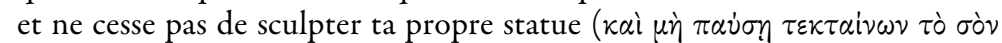
$\ddot{\alpha} \gamma \alpha \lambda \mu \alpha)$, jusqu'à ce que resplendisse pour toi la splendeur divine de la vertu, jusqu’à ce que tu voies la tempérance installée sur son socle sacré [Phèdre, 254b7]. Si tu es devenu cela, si tu l'as vu et si tu es resté avec toi-même de façon pure, sans avoir rien qui t'empêche d'être un de cette manière et sans avoir quelque chose d'autre qui soit mélangé à l'intérieur avec toi, mais si tu es toi-même tout entier uniquement une lumière véritable $[. .$.$] si tu te vois$ toi-même devenu cela, désormais devenu vision, ayant pris confiance en toi-même, même ici-bas étant dès à présent remonté, sans plus avoir besoin de guide, tends ton regard et vois; en effet, seul cet œil-là voit la grande beauté ${ }^{81}$.

Essayons de lire ce texte avec les yeux de Foucault, en imaginant les effets qu'en pourrait tirer son talent pour la transposition. Une grande part des traits du souci de soi hellénistico-romain trouverait sans peine un support expressif dans l'image plotinienne : la conversion vers soi-même ; l'explicitation du rapport à soi à travers une technique, la sculpture; l'alliance de la dimension morale à la dimension esthétique ${ }^{82}$; l'extension temporelle

81. Enn. I 6 [1] 9, 6-25, trad. Darras-Worms 2007 légèrement modifiée.

82. Alliance qui compte pour beaucoup dans la riche postérité de ce traité plotinien. À ce propos, Erwin Panofsky rappelle l'impact que cette image plotinienne a eu sur Michel- 
de l'injonction (《 ne cesse pas de sculpter ... ») au-delà de toute période déterminée; la dimension injonctive, qui saisit le rapport à soi mais qui pourrait tout aussi bien s'interpréter dans le rapport du maître ou du directeur de conscience au sujet; la transformation de soi, sous la figure, non d'une rupture ou d'un renoncement à soi, mais d'une épuration qui sépare le soi de ce qui n'est pas lui pour l'unir plus fortement à lui-même, pour intensifier son rapport à lui-même; l'unification, qui élimine du rapport à soi toute la multiplicité des éléments étrangers. Bien sûr, Foucault y relèverait sans doute aussi des traits du modèle platonicien : la conversion vers soi est inséparablement une «remontée » dans l'intelligible. Mais ces traits sont bien moins nombreux que ceux qui rendent l'image appropriable à d'autres formes de rapport à soi que la forme platonicienne.

Tout ou presque est dans cette image, donc. Mais l'image n'est pas dans L'Herméneutique du sujet. Une explication possible me semble être que la statue implique le rapport au divin. Or c'est là un thème qui, pour Foucault, appartient au modèle platonicien, ce qui n'a rien de surprenant, mais c'est aussi un thème qu'il veut n'appartenir qu'au modèle platonicien : il le minimise autant que possible dans les textes de l'époque impériale ${ }^{83}$. Aussi, projeter l'image de la sculpture de soi sur ces textes, en faire un schème pour penser le rapport à soi hellénistico-romain, ce serait nuire à cette stratégie de minimisation du rapport entre le soi et le divin dans le stoïcisme ou l'épicurisme, stratégie qui va de pair avec un effort pour faire refluer ce thème du divin sur le seul modèle platonicien. De fait, dans les quelques textes stoïciens qui utilisent le thème de la statue, même lorsque c'est avec une intention différente de celle de Plotin, il y a une constante : là où il est question de statue, le dieu n'est jamais loin. On l'a déjà vu dans le texte de Sénèque : se façonner soi-même, non dans de l'or ou de l'argent mais dans des matières plus modestes, c'est chercher à produire en soi ce bien inaliénable qu'est une âme vertueuse, c'est-à-dire un dieu dans un corps humain. Quelques références confirmeront cette solidarité entre l'image de la statue ou de la sculpture et le thème du rapport entre l'homme et le divin.

Ange, qui, à travers l'injonction à «sculpter sa propre statue », a trouvé chez Plotin « l'interprétation moralisante des procédés de la statuaire dans le sens d'une libération et d'une purification de soi par soi », pour l'appliquer à l'œuvre d'art; $c f$. Panofsky 1984, p. 91 , note 59 .

83. Cf. Foucault 2001, p. 401-402 : mentionnant la caractéristique du modèle platonicien qui place l'aboutissement de la connaissance de soi dans « la reconnaissance de soi comme élément divin », Foucault signale que le « principe de l'homoiosis to(i) theo(i) [...] est présent chez les stoïciens »; mais c'est aussitôt pour souligner qu'il «n'a pas la place centrale que l'on trouve dans le platonisme et dans le néoplatonisme ». Plus loin, le rapport entre l'âme et Zeus chez Épictète est distingué d'une assimilation et ramené à un « regard sur soi-même qui est en position d'analogie par rapport à ce qui constitue l'être divin » (p. 441). 
Dans sa Lettre 92 à Lucilius, Sénèque fait une distinction entre l'homme de bien et celui qui n'est pas encore parfait. C'est à propos de ce dernier, et du travail qu'il accomplit sur lui-même pour se perfectionner, qu'il recourt à un vocabulaire lié à la statuaire. Celui qui peut faillir « n'est pas encore homme de bien; il se façonne pour le devenir » (\$29); or, se façonner pour devenir homme de bien, c'est s'efforcer d'atteindre l'état de celui qui

se fait l'égal des dieux, <qui> aspire à l'être, car il se souvient de son origine. Ce n'est pas témérité que de vouloir remonter au lieu d'où l'on était descendu. Comment ne pas reconnaître quelque chose de divin chez un être qui est une partie du dieu ? $(\$ 30)$

La Lettre 41, également, passe de l'image de la statue à l'idée que le divin est en nous, et qu'il s'agit de le retrouver en nous, de prendre conscience de sa présence. Après avoir rappelé qu'il est inutile d'appeler de nos vœux la sagesse vu qu'elle est un bien que nous pouvons obtenir par nous-mêmes si nous nous y appliquons suffisamment, Sénèque exhorte Lucilius en ces termes :

Il ne s'agit pas d'élever les mains vers le ciel, de décider un sacristain à nous laisser arriver jusqu'à l'oreille de la statue [...] ; le dieu est près de toi ; il est avec toi ; il est en toi. Oui, Lucilius, un auguste esprit réside à l'intérieur de nous-mêmes.

Chez Épictète enfin, l'une des deux seules références à la statuaire qui ne soient ni insignifiantes ni négatives ${ }^{84}$ est là pour rappeler à son interlocuteur qu'il porte un dieu en lui, et qu'il est lui-même comme une statue façonnée par un dieu. Se rendre digne de son divin sculpteur doit imprégner la moindre de ses activités et norme la façon dont il a à s'acquitter de tous ses devoirs - lorsqu'il se nourrit, lorsqu'il se marie, lorsqu'il discute (exemples classiques de kathekonta chez Épictète), en un mot lorsqu'il s'exerce :

Tu es un fragment de Dieu; tu as en toi-même une part de divinité. Pourquoi donc ignorer la noblesse de ta naissance ? Comment ne sais-tu pas d'où tu viens? Ne veux-tu pas songer, quand tu manges, à l'être qui mange, à l'être que tu nourris ? dans tes rapports avec une femme, à l'être qui a ces rapports ? Dans la conversation, dans l'exercice, dans la discussion, ne sais-tu pas que tu nourris un dieu, que tu exerces un dieu ? Tu portes un dieu, [...] ce Dieu qui est intérieur à toi-même. [...] Si tu étais une statue

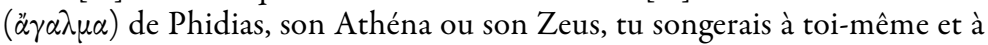
l'artiste qui t'a fait ; et, si tu prenais conscience, tu t'efforcerais de ne com-

84. L'autre de ces deux références est Entretiens, II 19, 23-25, texte cité supra n. 65. 
mettre aucun acte indigne de ton auteur ni de toi-même. [...] Laissez-moi seulement prendre assurance; vous verrez alors le regard et l'attitude qu'il faut; je vous ferai voir ce qu'est une statue lorsqu'elle est achevée et bien

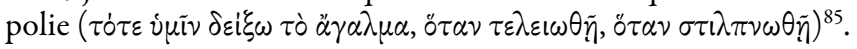

Quelle conclusion peut-on tirer de l'étude de ces quelques textes, qui tous mobilisent l'image de la statuaire pour exprimer le rapport de soi, non pas simplement à soi-même, mais au dieu intérieur ? Ils nous ramènent au caractère sélectif de la lecture foucaldienne, qui est à la fois le symptôme et l'outil de son projet d'histoire du sujet occidental. Les absents et les présents discrets de L'Herméneutique du sujet en disent autant sur ce projet que les figures de premier plan. Car ce sont les absents et les présents discrets qui sont la première condition de la mise en relief, de la production des contrastes : ils servent de moteur secret à la « dramatisation des distinctions conceptuelles $\gg^{86}$. L'étude de la place, ou plutôt de l'effacement du néoplatonisme dans le cours de 1982 concorde parfaitement avec les analyses, par exemple, de Carlos Lévy sur les sceptiques ${ }^{87}$, absents de L'Herméneutique du sujet, ou de Laurent Jaffro sur la notion de prohairesis ${ }^{88}$, absente des analyses pourtant abondantes et soignées de Foucault sur Épictète. Quel rôle joue le néoplatonisme, et particulièrement celui de Plotin, ce néoplatonisme dont il est si peu question dans L'Herméneutique du sujet? Le rôle d'absorber toute la dimension du rapport au divin, de figurer à lui seul l'intrication du rapport à soi et du rapport au divin dans la philosophie antique non chrétienne. Dès lors que cette intrication est voulue comme un trait constitutif du modèle platonicien, elle est dissociée du modèle qu'est le souci de soi romain - elle doit en être dissociée, coûte que coûte. Quitte pour cela à renoncer à la métaphore de la sculpture de soi, si séduisante, mais porteuse d'effets indésirables, brouillant les contours, défaisant les modèles. 


\section{BIBLIOGRAPHIE}

BÉNATOUïL, Th. 2003 : «Deux usages du stoïcisme : Deleuze, Foucault », dans Gros \& Lévy (éd.) 2003, p. 17-49.

BRÉHIER, É. (trad.) 1962 : Épictète, Entretiens, dans : Les Stoïciens, textes traduits par É. Bréhier, édités sous la direction de P.-M. Schuhl, Paris, 1962 (Bibliothèque de la Pléiade).

BRUNSCHWIG, J. 1998 : «La philosophie à l'époque hellénistique », dans M. CantoSperber (éd.), Philosophie grecque, Paris, ${ }^{2} 1998$, p. 457-591.

DARRAS-WormS, A.-L. (trad.) 2007 : Plotin, Traité 1 (I, 6), Introduction, traduction, commentaire et notes, Paris, 2007 (Les Écrits de Plotin).

Festugière, A.-J. 1971 : «L'ordre de lecture des dialogues de Platon aux V V'-VI $I^{\mathrm{e}}$ siècles », Études de philosophie grecque, Paris, 1971, p. 535-550 [= Museum Helveticum, 26 (1969), p. 281-296].

Foucault, M. 1984a : Histoire de la sexualité, 2. L'Usage des plaisirs, Paris, 1984 (Bibliothèque des histoires).

- 1984b : Histoire de la sexualité, 3. Le Souci de soi, Paris, 1984 (Bibliothèque des histoires).

- 2001: L'Herméneutique du sujet. Cours au Collège de France (1981-1982), édition établie par Fr. Gros sous la direction de Fr. Ewald et A. Fontana, Paris, 2003 (Hautes Études).

Goulet-Cazé, M.-O. (éd.) 1999 : Diogène Laërce, Vies et doctrines des philosophes illustres, Paris, 1999 (La Pochothèque. Classiques modernes).

GROS, F. 2001 : « Situation du cours », dans Foucault 2001, p. 489-526.

- 2003 : «Introduction », dans Gros \& Lévy (éd.) 2003, p. 7-13.

GROS, F. \& C. LÉVY (éd.) 2003 : Foucault et la philosophie antique : actes du colloque international du 21-22 juin 2001, Paris, 2003 (Philosophie en cours).

Hadot, P. 1963 : Plotin ou la simplicité du regard, Paris, 1963 (La Recherche de l'absolu, 10).

- 1993a: «Un dialogue interrompu avec Michel Foucault. Convergences et divergences », dans P. Hadot, Exercices spirituels et philosophie antique, Paris, ${ }^{3} 1993$, p. 229-233.

- 1993b : «Exercices spirituels », dans Exercices spirituels et philosophie antique, Paris, ${ }^{3} 1993$, p. 13-58.

JAFFrO, L., 2003 : «Foucault et le stoïcisme. Sur l'historiographie de L'Herméneutique du sujet $\gg$, dans Gros \& Lévy (éd.) 2003, p. 51-83.

LÉVY, C. 2003 : « Michel Foucault et le scepticisme : réflexions sur un silence », dans Gros \& Lévy (éd.) 2003, p. 119-135.

Meunier, M. 1933 : Marc-Aurèle, Pensées pour moi-même suivies du Manuel d'Épictète et du Tableau de Cébès, traduction nouvelle avec prolégomènes et notes, Paris, 1933 (Classiques Garnier).

Mouze, L. 2007 : Platon, Phèdre, présentation par D. Babut, introduction, traduction, notes et bibliographie par L. Mouze, Paris, 2007 (Classiques de poche).

Noblot, H. 1993 : Sénèque, Lettres à Lucilius, traduction par H. Noblot révisée par P. Veyne, dans : Sénèque, Entretiens, Lettres à Lucilius, éd. établie par P. Veyne, Paris, 1993 (Bouquins).

PANOFSKY, Erwin, 1984 : Idea. Contribution à l'histoire du concept de l'ancienne théorie de l'art, trad. fr. Henri Joly, Paris, 1984 (Collection Idées, 490). 